\title{
Tests d'adéquations lisses pour la loi de Newcomb-Benford
}

\section{Smooths Tests of Goodness-of-fit for the Newcomb-Benford distribution}

\section{Gilles R. DUCHARME ${ }^{1}$,Samuel KACl ${ }^{1}$, Credo VOVOR-DASSU ${ }^{1}$}

${ }^{1}$ IMAG, Univ. Montpellier, CNRS, Montpellier, France

Réception : 28/02/2020

Acceptation : 19/05/2020

Publication en ligne : 22/05/2020

RÉSUMÉ. La loi de probabilité de Newcomb-Benford est de plus en plus utilisée dans les applications de la statistique, notamment en détection de fraude. Dans ces contextes, il importe de déterminer si un jeu de données est issu de cette loi de probabilité en contrôlant les risques d'erreur de Type I, soit de faussement identifier une fraude, et de Type II, soit de ne pas la détecter. L'outil statistique qui permet d'exécuter ce genre de tâche est le test d'adéquation. Pour la loi de Newcomb-Benford, le test d'adéquation le plus populaire est le test du khi-deux de Pearson dont la probabilité d'erreur de Type II est reconnue comme étant assez grande. En conséquence, d'autres tests ont été récemment introduits. Le but de ce travail est de proposer de nouveaux tests d'adéquation pour cette loi, basés sur le principe des tests lisses. Ces tests sont ensuite comparés aux meilleurs tests existants pour ce problème. II en ressort que nos propositions sont globalement préférables aux tests existants et pourraient être utilisées dans les applications, notamment en détection de fraude. Un package de R,BENFORDSMOOTHTEST, est disponible sur le site GitHub pour effectuer nos tests.

ABSTRACT. The Newcomb-Benford probability distribution is becoming very popular in many areas using statistics, notably in fraud detection. In such contexts, it is important to be able to determine if a data set arises from this distribution while controlling the risk of a Type I error, i.e. falsely identifying a fraud, and a Type II error, i.e. not detecting that a fraud occurred. The statistical tool to do this work is a goodness-of-fit test. For the Newcomb-Benford distribution, the most popular such test is Pearson's chi-square test whose probability of a Type II error is known to be large. Consequently, other tests have been recently introduced. The goal of the present work is to build new goodness-of-fit tests for this distribution, based on the smooth test principle. These tests are then compared to some of their competitors. It turns out that the proposals of the paper are globally preferable to existing tests and should be seriously considered in fraud detection contexts, among others. The R package BENFORDSMOOTHTEST is available on GitHub to compute the test statistics.

MOTS-CLÉS. Loi de Newcomb-Benford, test d'adéquation, détection de fraudes, test lisse.

KEYWORDS. Newcomb-Benford's distribution, goodness-of-fit tests, fraud detection, smooth test.

\section{Introduction}

La loi de Newcomb-Benford (LNB) (Newcomb, 1881; Benford, 1938) annonce que sous certaines conditions, le premier chiffre significatif $(P C S)$ d'une variable aléatoire continue positive $X, D=$ $P C S(X)$, a pour probabilité $\mathbb{P}[D=d]=\pi_{d}=\log _{10}[1+1 / d], d \in\{1,2, \ldots, 9\}$.

L'utilisation de cette loi de probabilité connaît une popularité grandissante dans de nombreux domaines, notamment en détection de fraudes fiscales (Nigrini, 1996), financières (Cerioli et al., 2018), comptables (Durtschi et al., 2004) et scientifiques (Hein et al., 2012). Elle est aussi utilisée comme modèle statistique dans des disciplines aussi variées que l'hydrologie (Drake et Nigrini, 2000), la volcanologie (Geyer et Martí, 2012), la sismologie (Sambridge et al., 2011) et pour l'étude du trafic de données internet (Arshadi et Jahangir, 2014), entre autres applications. Cette popularité émane d'une part, du fait qu'on la rencontre empiriquement très souvent dans les jeux de données réelles (Durtschi et al., 2004) correspondant à des données dites de «deuxième génération », soit le résultat d'opérations (produits, puissances, etc.) de données brutes. D’autre part, des raisons théoriques font qu'elle apparaît 
aussi dans de nombreux contextes (Posch, 2008), sinon exactement du moins en tant qu'approximation de la réalité. En outre, elle interpelle l'intuition parce que, contrairement à ce que l'on pourrait naïvement penser, $D$ n'apparaît pas avec une probabilité de $1 / 9 \simeq 0.111$. Cette dissonance cognitive vient de ce que les psychologues appellent le «biais d'équiprobabilité » (Lecoutre, 1992). Ce biais est un des facteurs faisant que les $P C S$ de nombres influencés par la pensée humaine sont plus proches de la loi uniforme sur $\{1,2, \ldots, 9\}$ que de la LNB (Hill, 1998; Gauvrit et al., 2017). En particulier un fraudeur voulant trafiquer un jeu de données va inconsciemment avoir tendance à uniformiser leur PCS. Cette discordance offre une prise permettant à des auditeurs de détecter les fraudes, et cet outil s'est avéré particulièrement efficace dans le cas de fraudes fiscales (Ausloos et al., 2017). Un site internet, le Benford online bibliography (Berger et al., 2015) recense la quasi-totalité des publications sur la LNB, autant les résultats théoriques que les applications.

Il est donc souvent impératif de pouvoir déterminer si un jeu de données se conforme à la LNB en contrôlant les risques d'erreurs de Type I et II. Dans le contexte de la détection de fraudes, ces erreurs correspondent à faussement suspecter une fraude (Type I) avec pour conséquence le coût d'une audition approfondie subséquente, soit de ne pas détecter un jeu de données trafiquées (Type II) qui mènera ensuite à la prise de décisions erronées. L'outil statistique pour effectuer cette tâche est un test d'adéquation statistique (goodness-of-fit test). Pour ce problème, le test d'adéquation de préférence (Morrow, 2014) a longtemps été le test du $\chi^{2}$ de Pearson, (Lesperance et al., 2016, eq.3). Si $n_{d}$ est le nombre de fois dans un jeu de $n$ données que $P C S=d$, alors le test s'effectue en calculant la statistique de test :

$$
\chi^{2}=\sum_{i=1}^{9} \frac{\left(n_{d}-n \pi_{d}\right)^{2}}{n \pi_{d}},
$$

qui obéit approximativement, si $n$ est grand, à la loi $\chi_{8}^{2}$ si l'hypothèse nulle $H_{0}$ voulant que les données suivent une LNB est juste. On rejette $H_{0}$ si $\chi^{2}$ dépasse le quantile de cette loi approprié au risque d'erreur de Type I souhaité. Ce test est simple d'utilisation mais sa puissance, qui vaut 1 moins la probabilité d'une erreur de Type II, n'est en général pas reconnue comme étant très élevée (Morrow, 2014). Ainsi d'autres tests d'adéquation ont été proposés, certains étant des adaptations de tests développés pour des données continues et basés sur des principes statistiques reconnus comme les tests de Carmer-von Mises ou de Watson dans (Lesperance et al., 2016), d'autres sur la base de considérations intuitives (Joenssen, 2014). Un certain nombre de ces tests (voir Section 3) sont disponibles dans le package R BENFORDTEST (Joenssen, 2013a).

Malgré l'importance de l'utilisation d'un «bon » test d'adéquation pour la détection de fraude, ce n'est que récemment (Morrow, 2014; Joenssen, 2014; Lesperance et al., 2016) que les premières analyses de la puissance de ces différents tests à la LNB ont été réalisées. La procédure pour comparer entre eux plusieurs tests d'adéquation est bien rodée : on détermine une liste d'hypothèses alternatives couvrant les écarts que l'on estime plausibles à $H_{0}$, on génère des pseudo-échantillons de chacune de ces alternatives, on applique les tests au même niveau, soit la probabilité d'une erreur de Type I (en général 0.05), puis on constate s'ils ont rejeté ou non $H_{0}$. En répétant ceci un grand nombre de fois, on obtient une approximation de la puissance des différents tests que l'on peut ensuite comparer entre eux. En général, aucun test n'est uniformément le plus puissant, chacun ayant ses forces et ses faiblesses en regard des alternatives considérées. Un «bon» test se range régulièrement parmi les tests les plus puissants. Dans ce contexte, l'introduction d'un nouveau test se justifie si on peut montrer qu'il se range aussi parmi 
les plus puissants pour des alternatives courantes, ou s'il est performant pour de nouvelles alternatives importantes qui n'avaient pas auparavant été considérées.

En détection de fraudes, il s'ajoute à cette procédure d'évaluation de la qualité d'un test d'adéquation une dimension supplémentaire. Maintenant que l'existence d'outils de détection basés sur la LNB est bien connue. un fraudeur astucieux va chercher à trafiquer les données de facon à passer inapercu (Gauvrit et al., 2017). En effet, la crainte d'être détecté est un puissant frein à la fraude (Abdullahi et Mansor, 2015). Si le fraudeur sait que l'auditeur de ses données trafiquées exploitera tel test d'adéquation, il essaiera de faire en sorte que ce test ne détecte pas d'écart à la LNB. Dans ce contexte, chaque nouveau test ajoute une contrainte supplémentaire augmentant la difficulté de sa tâche. En outre, avec plusieurs tests utilisés en batterie, l'auditeur a aussi le choix de moduler leur emploi d'une façon inconnue du fraudeur, augmentant ainsi les risques de détection. Dans ce contexte, l'introduction d'un nouveau test d'adéquation est justifiée non seulement par sa bonne puissance, mais aussi parce que son existence peut complexifier la tâche du fraudeur. En ce domaine, viser l'éradication de la fraude est quasi impossible; on cherche de façon plus réaliste à la rendre difficile pour aider à sa prévention Abdullahi et Mansor (2015).

La famille des tests lisses (smooth tests) introduite par Neyman (Neyman, 1937) s'applique à des données autant discrètes que continues. Ces tests sont plus complexes à développer, car ils sont spécifiques à la loi de probabilité postulée en $H_{0}$ mais au fil des années, leurs grandes qualités ont été reconnues et ceci a mené Rayner et Best (1990, p.9) à la recommandation : « Don't use those other methods-use a smooth test!». Une version plus moderne, pilotée dans les données, a été proposée par Ledwina (1994) et a conduit Inglot et al. (1994) à affiner cette recommandation en : «Use a data-driven smooth test ! . Mais jusqu'à présent, ces tests n'ont pas été développés pour tester l'adéquation à la LNB.

Le but du présent travail est de développer différentes variantes des tests lisses pour le cas de la LNB et d'en étudier les qualités. La Section 2 rappelle les éléments théoriques permettant de construire une stratégie de test lisse et donne l'expression des statistiques de test pour le cas de la LNB. Quelques variantes sont introduites ainsi que des résultats théoriques concernant les puissances. La suite du travail explore les avantages d'inclure ces tests lisses dans une procédure de détection de fraude. La Section 3 présente une liste assez exhaustive des tests d'adéquation compétiteurs aux tests lisses et précise ceux qui sont retenus par la suite. La Section 4 présente les alternatives qui sont considérées pour la comparaison des puissances des tests retenus. La Section 5 présente les résultats d'une expérience de simulation qui montre que l'introduction des tests lisses est tout-à-fait justifiée selon les critères évoqués plus haut.

\section{Test lisse pour la LNB}

Le théorème suivant explique comment construire une famille, indexée par l'entier $K$, de tests lisses d'adéquation pour l'hypothèse nulle $H_{0}: X \sim f(\cdot)$. Ce théorème est bien connu et une référence est Thas (2010) par exemple.

Théorème 2.1. Soit $X_{1}, \ldots, X_{n}$ des copies indépendantes d'une variable aléatoire $X$ de densité $f(\cdot)$ par rapport à une mesure $\nu$. Soit $\left\{h_{0}(\cdot) \equiv 1, h_{k}(\cdot), k=1,2, \ldots\right\}$ une suite de fonctions orthonormales par rapport à $f(\cdot)$; plus précisément, $\int h_{k}(x) h_{k^{\prime}}(x) f(x) d \nu(x)=\delta_{k k^{\prime}}$, la fonction delta de Kronecker. Soit $U_{k}=n^{-1 / 2} \sum_{i=1}^{n} h_{k}\left(X_{i}\right)$ et pour un entier $K \geq 1$, soit $T_{K}=\sum_{k=1}^{K} U_{k}^{2}$. Alors sous $H_{0}, T_{K} \stackrel{L}{\longrightarrow} \chi_{K}^{2}$, la loi khi-deux à $K$ degrés de liberté, et un test de niveau asymptotique $\alpha$ rejette $H_{0}$ si la valeur observée de $T_{K}$ dépasse $x_{K, 1-\alpha}^{2}$, le quantile d'ordre $1-\alpha$ de cette loi $\chi_{K}^{2}$. 
Nous spécialisons maintenant ce théorème au cas où $f(\cdot)$ est la densité de la LNB. Pour ce faire, il faut déterminer des fonctions orthonormales $h_{k}(\cdot)$. Comme tous les moments de la LNB existent, nous pouvons, à l'instar de Neyman (1937) et de nombreux autres auteurs par la suite, choisir des polynômes. Le théorème suivant est aussi connu (Boulerice et Ducharme, 1997). Dans la suite l'indice « 0 » dénote un opérateur probabiliste calculé sous $H_{0}: X \sim f(\cdot)$.

Théorème 2.2. Soit $\mu_{k}=\mathbb{E}_{0}\left(X^{k}\right), k \geq 0$. Soit aussi la matrice $\mathbf{M}_{k}=\left[\mu_{i+i^{\prime}}\right]_{i, i^{\prime}=0, \ldots, k-1}$, le vecteur $\boldsymbol{\mu}_{k}=\left(\mu_{k}, \mu_{k+1}, \ldots \mu_{2 k-1}\right)^{T}$ et la constante $c_{k}=\mu_{2 k}-\boldsymbol{\mu}_{k}^{T} \mathbf{M}_{k}^{-1} \boldsymbol{\mu}_{k}$. Alors les polynômes

$$
h_{k}(x)=c_{k}^{-1 / 2}\left(x^{k}-\left(1, x, x^{2}, \ldots, x^{k-1}\right) \mathbf{M}_{k}^{-1} \boldsymbol{\mu}_{k}\right)
$$

satisfont la condition du Théorème 2.1.

Les moments de la LNB ont des expressions explicites complexes. Il en va de même des coefficients des polynômes $h_{k}(\cdot)$ dont les expressions exactes sont très longues dès lors que $k>2$. C'est pourquoi il est préférable de les exprimer sous une forme approximative. Mais ces calculs doivent être faits avec soin, car si les approximations numériques sont effectuées au niveau des éléments du Théorème 2.2, il en découle des erreurs d'arrondis qui détruisent l'orthonormalité. Ainsi, les coefficients des $h_{k}(\cdot)$ doivent être calculés en valeurs exactes, puis convertis en approximations numériques. En utilisant le logiciel MATHEMATICA, on obtient ainsi :

$$
\begin{aligned}
& h_{1}(x)=-1.3979+0.4063 x \\
& h_{2}(x)=2.2836-1.6128 x+0.18247 x^{2} \\
& h_{3}(x)=4.0815+4.5719 x-1.2053 x^{2}+0.0862 x^{3} \\
& h_{4}(x)=8.0795-12.0946 x+5.1951 x^{2}-0.8249 x^{3}+0.0431 x^{4} \\
& h_{5}(x)=-18.1064+33.1385 x-19.7207 x^{2}+5.0168 x^{3}-0.5665 x^{4}+0.0233 x^{5}
\end{aligned}
$$

Soit $D_{1}, \ldots, D_{n}$ un échantillon aléatoire de $P C S$. Pour effectuer un test lisse de l'hypothèse nulle $H_{0}$ : $D \sim L N B$ au niveau asymptotique $\alpha$, il suffit de calculer la statistique $T_{K}$ avec les $D_{i}$ en lieu et place des $X_{i}$ et de confronter la valeur observée de cette statistique de test au quantile d'ordre $1-\alpha$ de la loi $\chi_{K}^{2}$. Le package de R BENFORDSmoothTEST, disponible sur le site GitHub, permet d'effectuer ce test lisse pour $K$ allant jusqu'à 7 .

Remarque 2.3. Dans le Théorème 2.1. l'approximation $\chi_{K}^{2}$ est basée sur une convergence quand $n \rightarrow$ $\infty$. Si $n$ est petit, $x_{K, 1-\alpha}^{2}$ peut donner une mauvaise approximation du quantile exact de la loi de $T_{K}$. S'il est nécessaire d'assurer un contrôle précis de l'erreur de Type I, on peut approcher la valeur exacte de ce quantile par la méthode de Monte-Carlo. C'est ce qui est fait dans le Package BENFORDSMOOTHTEST, dès lors que $n<100$.

Remarque 2.4. Soit $g(x)$ une alternative fixée à la LNB. La puissance du test basé sur $T_{K}$ peut être assez bien approximée par l'expression suivante qui se trouve dans Inglot et al. (1994. Théorème 2.1) et dont les conditions d'applicabilité sont rencontrées par virtuellement tous les $g(x)$ raisonnables dans le présent contexte. Soit $\nu_{k}=\sqrt{n} \sum_{x=1}^{9} h_{k}(x) g(x)$ regroupés dans $\boldsymbol{\nu}=\left(\nu_{1}, \ldots, \nu_{K}\right)^{T}$ et $\Sigma=$ $\left[\sigma_{i, j}\right]_{i, j=1, \ldots, K}$, où $\sigma_{i, j}=\sum_{x=1}^{9} h_{i}(x) h_{j}(x) g(x)-\nu_{i} \nu_{j} / n$. Posons la décomposition spectrale $\Sigma=\mathbf{P} \Lambda \mathbf{P}^{T}$ 
où $\Lambda=\operatorname{Diag}\left\{\lambda_{1}, \ldots, \lambda_{K}\right\}$ est la matrice des valeurs propres de $\Sigma$ et $\mathbf{P}$ et la matrice de ses vecteurs propres normalisés. Alors, uniformément en $t>0$,

$$
\mathbb{P}_{g}\left[T_{K}>t\right]=\mathbb{P}\left[\sum_{k=1}^{K} \lambda_{k} \chi_{1}^{2}\left(\delta_{k}^{2}\right)>t\right]+O\left(n^{-1 / 2}\right)
$$

où $\delta_{k}$ sont les composantes de $\Lambda^{-1 / 2} \mathrm{P} \nu$. Par ailleurs, pour approcher la loi de la somme pondérée des $\chi_{1}^{2}\left(\delta_{k}^{2}\right)$, on peut utiliser une approximation de Liu et al. (2009) commodément basée sur des moments. Adaptée au présent problème, cette approximation s'écrit :

$$
\mathbb{P}\left[\sum_{k=1}^{K} \lambda_{k} \chi_{1}^{2}\left(\delta_{k}^{2}\right)>x_{K, 1-\alpha}^{2}\right] \simeq \mathbb{P}\left[\chi_{\ell}^{2}\left(d^{2}\right)>\frac{a\left(x_{K, 1-\alpha}^{2}-c_{1}\right)}{\sqrt{c_{2}}}+(\ell+d)\right]
$$

où $\ell=a^{2}-2 d^{2}, d^{2}=\max \left[s_{1} a^{3}-a^{2}, 0\right], a=1 /\left(s_{1}-\sqrt{s_{1}^{2}-s_{2}}\right)$ si $s_{1}^{2}>s_{2}$ et $1 / s_{1}$ sinon, avec $s_{1}=c_{3} / c_{2}^{3 / 2}$ et $s_{2}=c_{4} / c_{2}^{2}$ avec $c_{i}=\operatorname{tr}\left(\Sigma^{i}\right)+i \nu^{T} \Sigma^{(i-1)} \nu, i=1, \ldots, 4$. Cette approximation donne en général de bons résultats dans le contexte de tests d'adéquation (Duchesne et Lafaye De Micheaux, 2010). L'existence d'une expression explicite approchant la puissance du test lisse permet de mieux comprendre comment évolue cette puissance en fonction de $g(x)$. En effet, pour plusieurs des tests concernant la LNB (voir Section 3), la seule façon de calculer la puissance est par simulations, ce qui ne permet pas une bonne étude de la sensibilité de cette puissance en regard des écarts entre $f(x)$ et $g(x)$.

Remarque 2.5. Le choix de l'hyperparamètre $K$ est un élément important de la stratégie d'un test lisse. De la Remarque 2.4 si $K$ est trop petit, certains couples $\left(\lambda_{k}, \delta_{k}^{2}\right)$ prenant de grandes valeurs n'apparaîtront pas dans la somme de l'équation (2) et le test perdra de la puissance car il ne tiendra pas compte de ces importants écarts à $H_{0}$; si K est trop grand, cette puissance est diluée par la considération de termes $\lambda_{k}$ ou $\delta_{k}^{2}$ nuls ou négligeables dans la statistique de test. Pour choisir judicieusement cet hyperparamètre, Ledwina (1994) a proposé une stratégie «data-driven » qui consiste à calculer, pour une valeur à choisir $K_{\max }$,

$$
\hat{K}=\underset{1 \leq k \leq K_{\max }}{\arg \max }\left\{T_{k}-k \log (n)\right\}
$$

et à utiliser la statistique de test $T_{\hat{K}}$ qui $\stackrel{L}{\longrightarrow} \chi_{1}^{2}$ sous $H_{0}$.

Ledwina (1994) explique que cette expression pour $\hat{K}$ découle de l'application de la procédure de sélection de modèles de Schwarz à une famille de densités emboîtant la loi postulée sous $H_{0}$. Une version locale $d u$ test $d u$ rapport de vraisemblances maximales mène ensuite à $T_{\hat{K}}$. La convergence vers la loi $\chi_{1}^{2}$ vient du fait que (Ledwina, 1994), sous $H_{0}, \hat{K} \stackrel{P r}{\longrightarrow} 1$. Ainsi le test basé sur $T_{\hat{K}}$ possède le niveau asymptotique visé et des p-values asymptotiques peuvent être obtenues. Quand $H_{0}$ ne tient pas, $\hat{K}$ converge vers une valeur de $k \leq K_{\max }$ telle que $\delta_{k}^{2}>0$. Or les $\delta_{k}^{2}$ non nuls sont de l'ordre $O(n)$ selon la Remarque 2.4 et comme $\log (n) / n \longrightarrow 0$, un résultat de Ducharme (1997, Sec. 3) assure que la probabilité à la droite de l'équation (2) converge vers 1 . Il en découle que le test basé sur $T_{\hat{K}}$ est consistant dès lors que les $\delta_{k}^{2}$ dans (2) ne sont pas nuls.

Le terme de pénalité log $(n)$ vient de l'application du critère bayésien de Schwarz. Quelques auteurs ont suggéré d'autres pénalités issues de critères alternatifs. Il a cependant été montré (Kallenberg, 2002) que le choix de n'importe quelle pénalité entre 2 et $\log (n)$ préserve le niveau et la consistance du test. Ainsi, la théorie ne permet pas un choix judicieux de cette pénalité. Par contre, de nombreuses simulations par 
Ledwina et ses coauteurs ont montré que la pénalité log $(n)$ est en général adéquate et que le test "data - driven » basé sur $T_{\hat{K}}$ est un bon compromis dans la famille de tests lisses $\left\{T_{k}, k=1, \ldots, K_{\max }\right\}$.

Enfin, on peut choisir $K_{\max }$ en exploitant de l'information contextuelle au problème (ce qu'on appelle le cas «horizon fini»), soit le laisser tendre vers $\infty$ ("horizon infini») à une vitesse qui dépend de $n$. Ledwina et ses coauteurs ont beaucoup travaillé sur cette vitesse et les résultats théoriques obtenus sont impressionnants, mais d'une utilité pratique limitée, car ils s'expriment sous la forme $K_{\max }=o\left(n^{j}\right)$ pour un $j$ dépendant du contexte du problème. Heureusement la puissance du test basé sur $T_{\hat{K}}$ en fonction de $K_{\max }$ plafonne rapidement, de sorte que le cas «horizon fini » avec $5 \leq K_{\max } \leq 7$ donne en général de bons résultats.

Remarque 2.6. Dans la famille des tests lisses $\left\{T_{k}, k=1, \ldots K_{\max }\right\}$, on retrouve souvent des statistiques déjà proposées dans la littérature sur la base de considérations intuitives. C'est le cas ici, où $T_{1} / 5.102674=\left(a^{*}\right)^{2}$, la statistique introduite par Judge et Schechter (2009) et que l'on retrouve dans le package $R$ BENFORDTEST (fonction MEANDIGIT.BENFTEST). Cette statistique est aussi reliée à la statistique du «Distortion Factor (DF) model » de Nigrini (1996).

\section{Les compétiteurs aux tests lisses}

Comme signalé dans l'introduction, le nombre de tests d'adéquation à la LNB est plutôt limité audelà du test du $\chi^{2}$ de Pearson (1) et ceci facilite la tâche des fraudeurs. Soit $D_{1}, \ldots, D_{n}$ un échantillon aléatoire de PCS. Pour $d=1, . ., 9$, dénotons par $\hat{p}_{d}=n_{d} / n$, la proportion de $d$ dans l'échantillon et $\pi_{d}$ les probabilités de la LNB. Soit aussi $S_{d}=\sum_{j=1}^{d} \hat{p}_{j}$ et $S_{d}^{*}=\sum_{j=1}^{d} \pi_{j}$. Posons $Z_{d}=S_{d}-S_{d}^{*}$ et $t_{d}=\left(\pi_{d}+\pi_{d+1}\right) / 2$, pour $d=1, . ., 8$ et $t_{9}=\left(\pi_{9}+\pi_{1}\right) / 2$. Lesperance et al. (2016) considèrent les versions discrètes des tests suivants basés sur les écarts entre la distribution cumulative empirique et la distribution cumulative théorique de la LNB :

$$
\begin{aligned}
& W_{n}^{2}=n \sum_{i=1}^{n} Z_{d}^{2} t_{d} \quad \text { (Cramer-von Mises), } \\
& U_{n}^{2}=n \sum_{i=1}^{n}\left(Z_{d}-\bar{Z}\right)^{2} t_{d}, \quad \text { (Watson) }
\end{aligned}
$$

dont une variante, dite de Freedman, se retrouve dans Joenssen (2014) et

$$
A^{2}=n \sum_{i=1}^{8} \frac{Z_{d}^{2} t_{d}}{T_{d}\left(1-T_{d}\right)} \quad \text { (Anderson-Darling). }
$$

Morrow (2014) considère la statistique de Kolmogorov $K_{n}=\sqrt{n} \max _{1 \leq d \leq 9}\left|S_{d}-S_{d}^{*}\right|$. Enfin, un certain nombre d'auteurs considèrent des tests basés sur les écarts entre les probabilités $\pi_{d}$ de la LNB et les $\hat{p}_{d}$. Leemis et al. (2000) proposent la statistique $m=\max _{1 \leq d \leq 9}\left|\hat{p}_{d}-\pi_{d}\right|$ alors que Cho et Gaines (2007) suggèrent $d=\sqrt{\sum_{d=1}^{9}\left(\hat{p}_{d}-\pi_{d}\right)^{2}}$ et Drake et Nigrini (2000) introduisent la «Mean Average Deviation » : $M A D=\frac{1}{9} \sum_{d=1}^{9}\left|\hat{p}_{d}-\pi_{d}\right|$. Enfin, dans un autre ordre d'idée, Judge et Schechter (2009) proposent la statistique $a^{*}=(\bar{D}-3.44027) /(5.55973)$ qui est liée à la statistique $T_{1}$ du test lisse (voir Remarque 2.6). Au meilleur de nos connaissances, cette nomenclature couvre pratiquement tous les tests existants à 
ce jour pour la LNB, sauf la statistique $J_{p}^{2}$ de Joenssen (2013b) inspirée du test de Shapiro-Wilks, qui ne sera pas considéré plus avant en raison de son mauvais comportement, un test adaptant la statistique de Hotelling qui semble peu intéressant car certains hyperparamètres doivent être choisis (sans critère précis à ce jour), et un test bayésien (Geyer et Williamson, 2004) qui ne permet pas le contrôle fréquentiste des erreurs de Type I et II, élément important pour les auditeurs.

Le package R BENFORDTESTS (version 1.2.0, 2015) maintenu par Joenssen (2013a) permet d'effectuer certains de ces tests via les fonctions CHISQ.BENFTEST (test du $\chi^{2}$ ), KS.BENFTEST (test de Kolmogorov), MDIST.BENFTEST (test basé sur $m$ ), EDIST.BENFTEST (test basé sur $d$ ), USQ.BENFTEST (variante de Freedman du test de Watson), JPSQ.BENFTEST (test basé sur $J_{p}^{2}$ ) et MEANDIGIT.BENFTEST (test basé sur $a^{*}$ ), auquels s'ajoute le test adaptant la statistique de Hotelling.

Signalons que Lesperance et al. (2016) et Joenssen (2014) recommandent le test de Watson ou sa variante de Freedman qui donnent de bons résultats dans leurs simulations dont nous reprenons certains éléments aux Sections 4 et 5, en ceci que leur puissance se range parmi les plus élevées pour les quelques alternatives qu'ils considèrent. Dans la suite de ce travail, nous comparons la puissance des présents tests lisses à celles de certains des tests plus haut sur une plage plus large d'alternatives. Plus précisément, comme représentant de la famille des tests lisses $\left\{T_{k}, k=1, \ldots K_{\max }\right\}$, nous retenons le test $T_{2}$ et sa version data-driven $T_{\hat{K}}$ (avec $K_{\max }=5$ ); comme représentant des tests basés sur les écarts entre distributions cumulatives, nous choisissons la statistique $U_{n}^{2}$ de Watson. Nous considérons le test MAD de Drake et Nigrini (2000) comme représentant des tests basés sur des écarts entre les $\pi_{d}$ de la LNB et les $\hat{p}_{d}$, auxquel nous ajoutons le test classique du $\chi^{2}$ de Pearson (1).

\section{Les alternatives}

Pour étudier la puissance des tests lisses de la Section 2 et la comparer à celle des tests retenus à la Section 3, nous devons préciser des alternatives pour lesquelles cette puissance sera calculée. Pour ce faire, nous considérons des familles d'alternatives indexées par un paramètre, génériquement noté $\beta$, et emboîtant la LNB. Notre première famille d'alternatives est celle de Rodriguez (2004) donnée par :

$$
\mathbb{P}[D=d]=p_{d}^{(R o d)}(\beta)= \begin{cases}\frac{1}{9}\left(1+\frac{10}{9} \ln (10)+x \ln (x)-(x+1) \ln (x+1)\right) & \text { si } \beta=0 \\ \log _{10}[1+1 / x] & \text { si } \beta=-1, \\ \frac{\beta+1}{9 \beta}-\left((x+1)^{(\beta+1)}-x^{(\beta+1)}\right) /\left(\beta\left(10^{(\beta+1)}-1\right)\right) & \text { sinon }\end{cases}
$$

pour $\beta \in \mathbb{R}$, où on peut remarquer que quand $\beta=0$, on retrouve la loi de Stigler Lee et al. (2010), $\beta=$ -1 donne la $\operatorname{LNB}$ et quand $\beta \rightarrow \pm \infty$, on a la loi uniforme discrète sur $\{1,2, \ldots, 9\}$, notée $U D[\{1, . ., 9\}]$. La deuxième famille d'alternatives est celle de la LNB généralisée de Pietronero et al. (2001) donnée par :

$$
\mathbb{P}[D=d]=p_{d}^{(\text {Piet })}(\beta)=\left\{\begin{array}{ll}
\frac{(d+1)^{(1-\beta)}-d^{(1-\beta)}}{10^{(1-\beta)}-1} & \text { si } \beta \neq 1 \\
\log _{10}[1+1 / x] & \text { si } \beta=1
\end{array},\right.
$$

où $\beta \in \mathbb{R}$ avec la LNB correspondant au cas $\beta=1$. La troisième famille de lois alternatives est celle de Hürlimann (2009) où

$$
\mathbb{P}[D=d]=p_{d}^{(\text {Hurl })}(\beta)=\frac{1}{2}\left(\log _{10}[1+x]^{\beta}-\log _{10}[x]^{\beta}-\left(1-\log _{10}[1+x]\right)^{\beta}+\left(1-\log _{10}[x]\right)^{\beta}\right),
$$


avec $\beta \in \mathbb{R}$. Notons que cette famille a la particularité de donner la LNB pour les deux valeurs $\beta=1,2$. La quatrième famille est celle d'un mélange de lois LNB et UD de la forme $(1-\beta) \times L N B+\beta \times$ $U D[\{0,1, \ldots, 9\}]$ avec $\beta \in[0,1]$, considérée par Lesperance et al. (2016). La cinquième famille de lois, aussi considérée par Lesperance et al. (2016), est celle d'une loi LNB contaminée où

$$
\mathbb{P}[D=d]=p_{d}^{(\text {conta-1) }}(\beta)=\left\{\begin{array}{ll}
\pi_{d} /(1+2 \beta) & \text { si } d \neq 1,9 \\
\left(\pi_{d}+\beta\right) /(1+2 \beta) & \text { si } d=1,9
\end{array},\right.
$$

où $\beta \geq 0$ et la LNB correspond au cas $\beta=0$. Enfin la dernière famille de lois est nouvelle; elle est obtenue en contaminant plus lourdement que la famille précédente l'aile de droite de la LNB :

$$
\mathbb{P}[D=d]=p_{d}^{(\text {conta-2) }}(\beta)=\left\{\begin{array}{ll}
\pi_{d} /(1+10 \beta) & \text { si } d=1, \ldots, 5 \\
\left(\pi_{d}+(d-5) \beta\right) /(1+10 \beta) & \text { si } d=6, \ldots, 9
\end{array},\right.
$$

où $\beta \geq 0$ et la LNB correspond au cas $\beta=0$. Ensemble, ces 6 familles couvrent de nombreuses alternatives à la LNB. Elles ont aussi été, pour les cinq premières, reconnues comme étant des alternatives plausibles, pouvant être rencontrées dans les applications. L'étude de la puissance des différents tests d'adéquation sur ces familles devrait donner une bonne idée de leur comportement en pratique. Terminons cette section en signalant que les cinq premières familles sont, à notre connaissance, les seules familles de lois existantes dans la littérature emboîtant la LNB.

\section{Simulations}

Dans cette section, nous étudions la puissance de différents tests d'adéquation à la LNB pour les alternatives de la Section 4 Notre but est de ranger les tests retenus à la Section 3 du meilleur au moins puissant, et ce pour chacune des six familles d'alternatives. Ceci permet de déterminer si les tests $T_{2}$ et $T_{\hat{K}}$ se rangent fréquemment parmi les meilleurs, justifiant ainsi leur introduction par le présent travail et leur utilisation en pratique. Pour chacune de ces familles d'alternatives, nous avons approché par simulation, selon la méthode exposée au paragraphe suivant, la courbe de puissance, en fonction de leur paramètre $\beta$, des tests retenus à la Section 3 pour des tailles d'échantillon variant entre $n=25$ et $n=5000$ et pour une large gamme de valeurs de $\beta$ dans le domaine des valeurs autorisées. En regardant attentivement ces courbes de puissances, nous avons constaté que dans chaque famille, l'ordre des fonctions de puissance des différents tests ne varie pas selon les tailles d'échantillon considérées. Ceci permet une réduction importante de l'analyse des résultats de la simulation : pour obtenir le rang des différents tests selon leur courbe de puissance, il suffit de sélectionner une seule taille d'échantillon par famille, laquelle servira de représentant, et d'exhiber les courbes de puissances des tests à cette taille. Nous avons choisi cette taille d'échantillon représentative de façon à ce que l'examen visuel soit aisé. La plage de valeurs de $\beta$ représentée a aussi été choisie pour une bonne lisibilité des graphiques et de façon à éviter les résultats triviaux (courbe de puissance trop proche de $\alpha=0.05$ et 1 ).

Ainsi pour la famille de Rodriguez, la taille d'échantillon représentative est $n=100$ avec $\beta \in[-4,2]$; pour celle de Pietronero, $n=25$ avec $\beta \in[-0.25,2.5]$; pour la famille de Hurlimann, $n=750$ avec $\beta \in[0.5,2.75]$; pour la famille de mélange de LNB et UD, $n=250$ avec $\beta \in[0.0,0.45]$. Enfin, pour les deux LNB contaminées, nous prenons $n=500$ avec $\beta \in[0,0.06]$ et $\beta \in[0.0,0.009]$ respectivement. Les tests sont effectués au niveau $5 \%$ et en regard de la Remarque 2.3, les quantiles de référence de tous les 
tests sont approximés par Monte Carlo (50 000 réplications) afin de permettre une juste comparaison des puissances. Les puissances des tests pour chaque triplet (famille, $n, \beta$ ) sont approximées par le nombre de rejets parmi 10000 réplications. Notons que pour le test $T_{2}$, les formules de la Remarque 2.4 sont aussi calculées et produisent des résultats remarquablement proches de ceux de la simulation.

Les graphiques des courbes de puissance des cinq tests retenus aux tailles d'échantillon représentatives apparaissent à la Figure 1, un panneau par famille d'alternatives. Comme les puissances de cette figure sont approximées de 10000 réplications, elles contiennent un bruit statistique que l'on peut évaluer à environ \pm 0.01 (au niveau de confiance $95 \%$ ) lorsque la puissance est autour de 0.5 . Ce point est pris en considération dans les conclusions qui suivent.
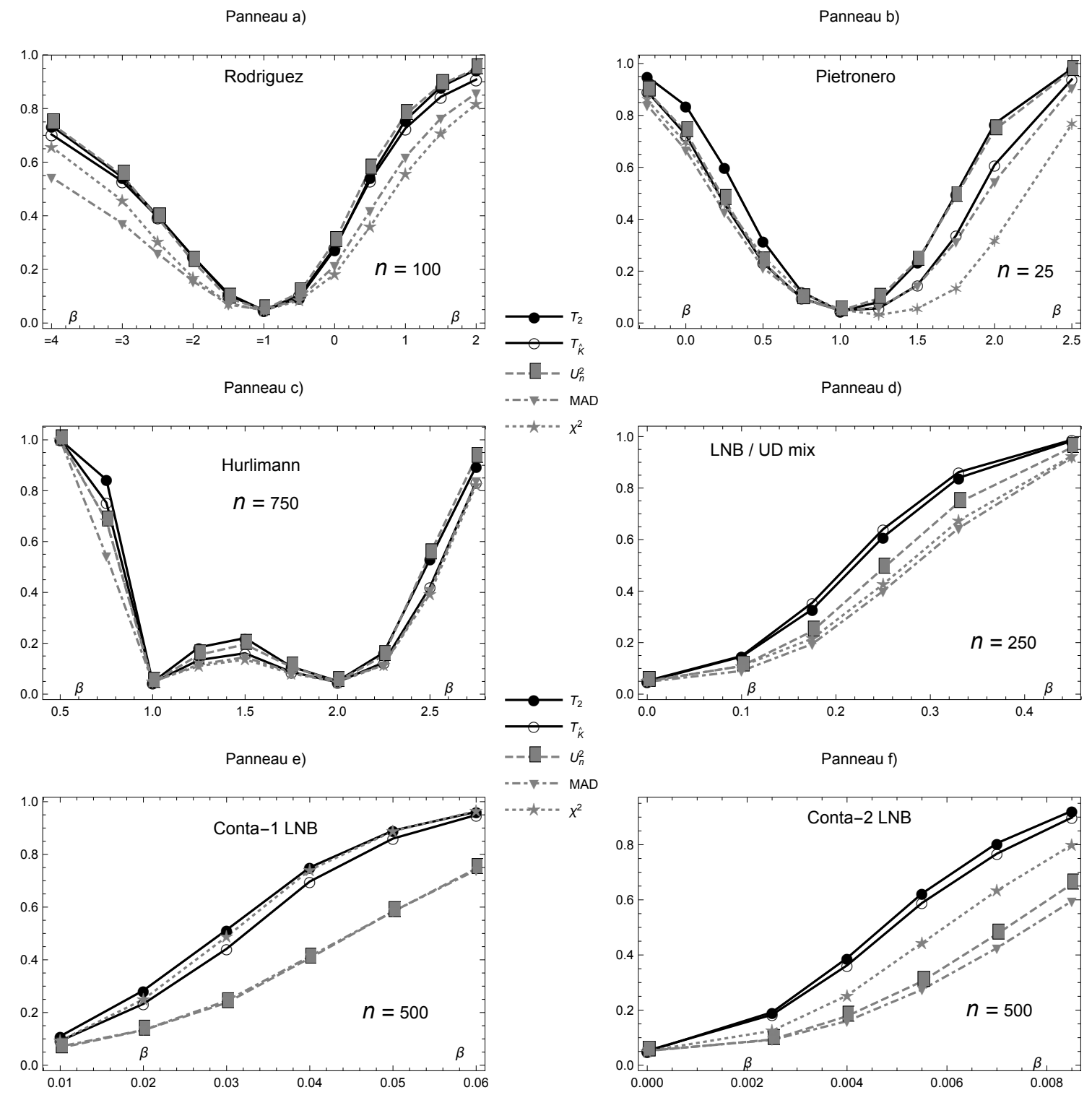

Figure 1: Courbes de puissance, en fonction du paramètre $\beta$, de divers tests (basés sur 10000 réplications) au niveau 5\% pour l'hypothèse nulle de loi LNB. Les familles d'alternatives sont décrites à la Section 4 . Les tests considérés sont $T_{2}$, (trait plein avec cercle plein), $T_{\hat{K}}$ (trait plein avec cercle vide), $U_{n}^{2}$ (tiret avec rectangle), MAD (tiret-pointillé avec triangle) et le test $\chi^{2}$ de Pearson (pointillé avec étoile), dont les expressions se trouvent à la Section 3. Les quantiles de référence sont approximés par Monte-Carlo en utilisant 50000 répétitions.

Pour les lois de Rodriguez du Panneau a), le test de Watson basé sur $U_{n}^{2}$ est comparable à $T_{2}$ et suivi de très près par $T_{\hat{K}}$. Les tests basés sur MAD et $\chi^{2}$ sont moins puissants (avec MAD meilleur que $\chi^{2}$ si $\beta>-1$ et inversement sinon). Pour les lois de Pietronero du Panneau b), $T_{2}$ est le test le plus puissant sur toute la plage de valeurs de $\beta$, suivi de $T_{\hat{K}}$ et de $U_{n}^{2}$ quand $\beta<1$ et inversement quand $\beta>1$. 
Encore là, les tests MAD et $\chi^{2}$ ferment la marche, comparables quand $\beta<1$ mais avec $\chi^{2}$ nettement moins puissant que MAD à droite. Pour la famille de Hurlimann du Panneau c), quand $\beta<1, T_{2}$ domine, suivi de $T_{\hat{K}}$ puis de $U_{n}^{2}$ alors que pour $\beta>1, T_{2}$ et $U_{n}^{2}$ sont comparables mais $T_{\hat{K}}$ est légèrement moins puissant. Encore là, MAD et $\chi^{2}$ ferment la marche. Pour la famille de mélange LNB et UD du Panneau d), $T_{\hat{K}}$ domine $T_{2}$, MAD et $\chi^{2}$ sont les moins puissants et $U_{n}^{2}$ a un comportement intermédiaire. Pour la première famille de lois contaminées du Panneau e), $T_{2}$ et $\chi^{2}$ dominent, suivi de près par $T_{\hat{K}}$ alors que $U_{n}^{2}$ et MAD ont une puissance nettement plus faible. Enfin pour la famille de lois contaminées du Panneau f), les deux tests lisses $T_{2}$ et $T_{\hat{K}}$ dominent nettement les autres, MAD et $U_{n}^{2}$ étant de loin inférieurs alors que $\chi^{2}$ a un comportement intermédiaire.

En conclusion, on retire de cette expérience de simulation que le test $T_{2}$ est toujours parmi les deux meilleurs tests en termes de puissance, disputant souvent la tête avec le test basé sur $T_{\hat{K}}$. Le test MAD est toujours parmi les deux plus mauvais et ne devrait pas être utilisé. Les tests du $\chi^{2}$ et $U_{n}^{2}$ ont des comportements plus variables. En regard de notre définition de «bon » test d'adéquation évoquée dans l'introduction, nous pouvons donc recommander l'un ou l'autre des tests $T_{2}$ ou $T_{\hat{K}}$. Leur introduction est ainsi justifiée et le fait qu'il s'agisse de tests nouveaux, augmentant l'arsenal des auditeurs, ajoute à leur utilité. Nous confirmons Morrow (2014) que le test du $\chi^{2}$ de Pearson est généralement inférieur . Enfin, nos résultats atténuent les conclusions de Lesperance et al. (2016) et Joenssen (2014) qui recommandent le test $U_{n}^{2}$ de Watson.

\section{Bibliographie}

R. Abdullahi and N. Mansor. Fraud triangle theory and fraud diamond theory. understanding the convergent and divergent for future research. International Journal of Academic Research in Accounting, Finance and Management Sciences, 5(4) : 38-45, 10 2015. doi: 10.6007/ijarafms/v5-i4/1823.

L. Arshadi and A. H. Jahangir. Benford's law behavior of internet traffic. Journal of Network and Computer Applications, 40 :194-205, 4 2014. ISSN 1084-8045. doi: 10.1016/j.jnca.2013.09.007.

M. Ausloos, R. Cerqueti, and T. A. Mir. Data science for assessing possible tax income manipulation : The case of italy. Chaos, Solitons \& Fractals, 104 :238-256, 11 2017. ISSN 0960-0779. doi: 10.1016/j.chaos.2017.08.012.

F. Benford. The law of anomalous numbers. Proceedings of the American Philosophical Society, 78(4) :551-572, 1938.

A. Berger, T. Hill, and E. Rogers. Benford online bibliography, 05 2015. URL http://www . benfordonline. net.

B. Boulerice and G. R. Ducharme. Smooth tests of goodness-of-fit for directional and axial data. Journal of Multivariate Analysis, 60(1) :154-175, 01 1997. doi: 10.1006/jmva.1996.1650.

A. Cerioli, L. Barabesi, A. Cerasa, M. Menegatti, and D. Perrotta. Newcomb-benford law and the detection of frauds in international trade. Proceedings of the National Academy of Sciences, 116(1) :106-115, dec 2018. ISSN 0027-8424. doi: 10.1073/pnas.1806617115.

W. T. Cho and B. Gaines. Breaking the (benford) law. The American Statistician, 61(3) :218-223, 8 2007. doi: 10.1198/ $000313007 \times 223496$.

P. D. Drake and M. J. Nigrini. Computer assisted analytical procedures using benford's law. Journal of Accounting Education, 18(2) :127-146, 3 2000. ISSN 0748-5751. doi: 10.1016/s0748-5751(00)00008-7.

G. R. Ducharme. Consistent selection of the actual model in regression analysis. Journal of Applied Statistics, 24(5) : 549-558, oct 1997. doi: 10.1080/02664769723530.

P. Duchesne and P. Lafaye De Micheaux. Computing the distribution of quadratic forms : Further comparisons between the liu-tang-zhang approximation and exact methods. Computational Statistics \& Data Analysis, 54(4) :858-862, 4 2010. doi: 10.1016/j.csda.2009.11.025.

C. Durtschi, H. William, and P. Carl. The effective use of benford's law to assist in detecting fraud in accounting data. Journal Forensic Account, 5 :17-34, 01 2004. ISSN 1524-5586. 
N. Gauvrit, J.-C. Houillon, and J.-P. Delahaye. Generalized benford's law as a lie detector. Advances in Cognitive Psychology, 13(2) :121-127, jun 2017. doi: 10.5709/acp-0212-x.

A. Geyer and J. Martí. Applying benford's law to volcanology. Geology, 40(4) :327-330, 04 2012. ISSN 0091-7613. doi: 10.1130/G32787.1.

C. L. Geyer and P. P. Williamson. Detecting fraud in data sets using benford's law. Communications in Statistics - Simulation and Computation, 33(1) :229-246, jan 2004. doi: 10.1081/sac-120028442.

J. Hein, R. Zobrist, C. Konrad, and G. Schuepfer. Scientific fraud in 20 falsified anesthesia papers. Der Anaesthesist, 61 (6) :543-549, jun 2012. doi: 10.1007/s00101-012-2029-x.

T. P. Hill. The first digit phenomenon : A century-old observation about an unexpected pattern in many numerical tables applies to the stock market, census statistics and accounting data. American Scientist, 86(4) :358-363, 1998. ISSN 00030996.

W. Hürlimann. Generalizing benford's law using power laws : Application to integer sequences. International Journal of Mathematics and Mathematical Sciences, 2009 :1-10, 07 2009. doi: 10.1155/2009/970284.

T. Inglot, W. Kallenberg, and T. Ledwina. Power approximations to and power comparison of smooth goodness-of-fit tests. Scandinavian journal of statistics, (21):131-145, 1994. ISSN 0303-6898.

D. W. Joenssen. Statistical tests for evaluating conformity to benford's law. The Comprehensive R Archive Network, 2013 a. URL https: //cran.r-project.org/package=BenfordTests.

D. W. Joenssen. A new test for benford's distribution. Abstract-Proceedings of the 3rdJoint Statistical Meeting DAGStat, $032013 b$.

D. W. Joenssen. Testing for benford's law : A monte carlo comparison of methods. SSRN Electronic Journal, 11 2014. doi: $10.2139 /$ ssrn.2545243.

G. Judge and L. Schechter. Detecting problems in survey data using benford's law. Journal of Human Resources, 44(1) : 1-24, 2009. doi: 10.3368/jhr.44.1.1.

W. Kallenberg. The penalty in data driven neyman's tests. Mathematical methods of statistics, $11: 323-340,2002$. ISSN 1066-5307.

W. C. Kallenberg and L. Teresa. Data driven smooth tests for composite hypotheses comparison of powers. Journal of Statistical Computation and Simulation, 59(2) :101-121, oct 1997. doi: 10.1080/00949659708811850.

M.-P. Lecoutre. Cognitive models and problem spaces in ?purely random? situations. Educational Studies in Mathematics, 23(6) :557-568, dec 1992. doi: 10.1007/bf00540060.

T. Ledwina. Data-driven version of neyman's smooth test of fit. Journal of the American Statistical Association, 89(427) : 1000-1005, sep 1994. doi: 10.1080/01621459.1994.10476834.

J. Lee, W. K. T. Cho, and G. G. Judge. Stigler's approach to recovering the distribution of first significant digits in natural data sets. Statistics \& Probability Letters, 80(2) :82-88, jan 2010. doi: 10.1016/j.spl.2009.09.015.

L. M. Leemis, B. W. Schmeiser, and D. L. Evans. Survival distributions satisfying benford's law. The American Statistician, 54(4) :236-241, nov 2000. doi: 10.1080/00031305.2000.10474554.

M. Lesperance, W. J. Reed, M. A. Stephens, C. Tsao, and B. Wilton. Assessing conformance with benford's law : Goodnessof-fit tests and simultaneous confidence intervals. PLOS ONE, 11(3) :e0151235, mar 2016. doi: 10.1371/journal.pone. 0151235 .

H. Liu, Y. Tang, and H. H. Zhang. A new chi-square approximation to the distribution of non-negative definite quadratic forms in non-central normal variables. Computational Statistics \& Data Analysis, 53(4) :853-856, feb 2009. ISSN 01679473. doi: 10.1016/j.csda.2008.11.025.

J. Morrow. Benford's Law, Families of Distributions and a Test Basis. Centre for Economic Performance, LSE, (dp1291), Aug. 2014.

S. Newcomb. Note on the Frequency of Use of the Different Digits in Natural Numbers. American Journal of Mathematics, 4 :39-40, Jan. 1881. ISSN 00029327.

J. Neyman. »smooth test» for goodness of fit. Scandinavian Actuarial Journal, 1937(3-4) :149-199, jul 1937. doi: 10.1080/03461238.1937.10404821.

M. J. Nigrini. A taxpayer compliance application of benford's law. The Journal of the American Taxation Association, 18 (1) :72-91, 04 1996. ISSN 0198-9073. 
M. J. Nigrini and S. J. Miller. Benford's law applied to hydrology data-results and relevance to other geophysical data. Mathematical Geology, 39(5) :469-490, aug 2007. doi: 10.1007/s11004-007-9109-5.

L. Pietronero, E. Tosatti, V. Tosatti, and A. Vespignani. Explaining the uneven distribution of numbers in nature : the laws of benford and zipf. Physica A : Statistical Mechanics and its Applications, 293(1-2) :297-304, apr 2001. ISSN 0378-4371. doi: 10.1016/s0378-4371(00)00633-6.

P. N. Posch. A survey on sequences and distribution functions satisfying the first-digit-law. Journal of Statistics and Management Systems, 11(1) :1-19, jan 2008. doi: 10.1080/09720510.2008.10701294.

J. C. W. Rayner and D. J. Best. Smooth tests of goodness of fit : An overview. International Statistical Review / Revue Internationale de Statistique, 58(1) :9, apr 1990. ISSN 03067734, 17515823. doi: 10.2307/1403470.

R. J. Rodriguez. First significant digit patterns from mixtures of uniform distributions. The American Statistician, 58(1) : 64-71, feb 2004. doi: 10.1198/0003130042782.

M. Sambridge, H. Tkalcic, and P. Arroucau. Benford's law of first digits : From mathematical curiosity to change detector. Asia Pacific Mathematics Newsletter(APMN), $1: 1-5,012011$.

O. Thas. Comparing Distributions. Springer New York, 1 edition, 2010. ISBN 978-0-387-92710-7. doi: 10.1007/ 978-0-387-92710-7. 